\title{
ON THE RELATION BETWEEN THE ABEL-TYPE AND BOREL-TYPE METHODS OF SUMMABILITY
}

\author{
B. L. R. SHAWYER AND G. S. YANG ${ }^{1}$
}

ABSTRACT. In recent papers, R. Jajte and B. L. R. Shawyer have proved that under certain conditions a series summable by $a$ Borel-type or absolute Borel-type method of summability is also summable by the Abel or absolute Abel method of summability to the same sum. In the present paper, the Abel method is replaced by the more general Abel-type method, giving similar results for ordinary, strong and absolute summability.

1. Introduction. It is known that the Abel method and the Borel exponential method of summability are not equivalent, but that under certain conditions, both methods sum the same series to the same sum [4]. This was recently extended in one direction, to the conditions under which a series summable by a Borel-type method is also summable by the Abel method [5]. The last result was extended to absolute summability by one of the present authors [9]. The object of this paper is to replace the Abel method by the more general Abel-type method and give results for ordinary, strong and absolute summability.

2. Definitions. Suppose throughout that $\sigma, a_{n}(n=0,1, \cdots)$ are arbitrary complex numbers, that $\lambda>-1$, that $\alpha>0$ and that $\beta$ is real. Let $N$ be any nonnegative integer greater than $1-\beta / \alpha$.

Define

$$
s_{n}=\sum_{r=0}^{n} a_{r} ; \quad s_{-1}=0 .
$$

2.1 Definitions of the Borel-type methods of summability. Define

$$
s(x)=\sum_{n=N}^{\infty}\left(s_{n} x^{\alpha n+\beta-1}\right) / \Gamma(\alpha n+\beta) ; \quad S_{\alpha, \beta}(x)=\alpha e^{-x} s(x) .
$$

This series is assumed convergent for all $x \geqq 0$.

Ordinary Summability [1]. If $S_{\alpha, \beta}(x) \rightarrow \sigma$ as $x \rightarrow \infty$, then $s_{n} \rightarrow \sigma(B, \alpha, \beta)$.

Received by the editors January 12, 1970.

AMS 1969 subject classifications. Primary 4030, 4033.

Key words and phrases. Ordinary, strong, absolute, Abel-type summability methods, Borel-type summability methods.

1 The research in this paper was supported in part by the National Research Council of Canada. 
Strong Summability [3]. If

$$
\int_{0}^{x} e^{t}\left|S_{\alpha, \beta-1}(t)-\sigma\right|^{p} d t=o\left(e^{x}\right) \quad(p \geqq 1)
$$

as $x \rightarrow \infty$, then $s_{n} \rightarrow \sigma[B, \alpha, \beta]_{p}$.

Absolute Summability [3]. If $S_{\alpha, \beta}(x) \rightarrow \sigma$ as $x \rightarrow \infty$ and $S_{\alpha, \beta}(x)$ is of bounded variation with respect to $x$ in the range $[0, \infty)$, then $s_{n} \rightarrow \sigma|B, \alpha, \beta|$.

2.2 Definitions of the Abel-type methods of summability. Define

$$
\begin{aligned}
E_{n}^{\lambda} & =\left(\begin{array}{c}
\lambda+n \\
n
\end{array}\right)=\frac{(\lambda+1)(\lambda+2) \cdots(\lambda+n)}{n !} \text { for } n=0,1, \cdots, \\
E_{0}^{\lambda} & =1, \quad E_{n}^{\lambda}=0 \text { for } n=-1,-2, \cdots, \\
\sigma_{\lambda}(y) & =(1+y)^{-\lambda-1} \sum_{n=0}^{\infty} E_{n}^{\lambda} s_{n}\left(\frac{y}{1+y}\right)^{n}, \quad y>0 .
\end{aligned}
$$

ORdinaRy Summability [2]. If the series defining $\sigma_{\lambda}(y)$ is convergent for all $y>0$ and $\sigma_{\lambda}(y) \rightarrow \sigma$ as $y \rightarrow \infty$, then $s_{n} \rightarrow \sigma\left(A_{\lambda}\right)$.

Strong Summability [6]. If the series defining $\sigma_{\lambda}(y)$ is convergent for all $y>0$, and

$$
\int_{0}^{y}\left|\sigma_{\lambda}(t)-\sigma\right|^{p} d t=o(y) \quad(p \geqq 1) \quad \text { as } y \rightarrow \infty,
$$

then $s_{n} \rightarrow \sigma\left[A_{\lambda-1}\right]_{p}$.

Absolute Summability [7]. If the series defining $\sigma_{\lambda}(y)$ is convergent for all $y>0, s_{n} \rightarrow \sigma\left(A_{\lambda}\right)$ and $\sigma_{\lambda}(y)$ is of bounded variation with respect to $y$ in the range $[0, \infty)$, then $s_{n} \rightarrow \sigma\left|A_{\lambda}\right|$.

Note. The condition that

$$
\sum_{n=0}^{\infty} a_{n} x^{n} \text { is convergent for all } x \text { in }(-1,1)
$$

will be a condition for each main theorem of this paper. It can easily be shown that the condition (C) implies that the series defining $\sigma_{\lambda}(y)$ is convergent for all $y>0$.

3. Preliminary results. The following lemmas are required:

LEMMA 1. If

$$
F(w)=\int_{a}^{b} g(w, u) f(u) d u \quad(w \geqq h>0,-\infty<a<b<\infty),
$$


and

$$
\int_{h}^{\infty}\left|d_{w} g(w, u)\right| \leqq g(u) \quad(a<u<b),
$$

then

$$
\int_{h}^{\infty}|d F(w)| \leqq \int_{a}^{b} g(u)|f(u)| d u
$$

Proof. Let $h=w_{0}<w_{1}<\cdots<w_{m}$, then for all $m$

$$
\begin{aligned}
\sum_{k=0}^{m-1}\left|F\left(w_{k+1}\right)-F\left(w_{k}\right)\right| & \leqq \int_{a}^{b} \sum_{k=0}^{m-1}\left|g\left(w_{k+1}, u\right)-g\left(w_{k}, u\right)\right||f(u)| d u \\
& \leqq \int_{a}^{b} g(u)|f(u)| d u .
\end{aligned}
$$

Hence

$$
\int_{h}^{\infty}|d F(w)| \leqq \int_{a}^{b} g(u)|f(u)| d u .
$$

LemmA 2. Suppose that $m$ is a positive integer and that

$$
\mu_{n}=\prod_{i=1}^{m} \frac{b_{i} n+c_{i}}{d_{i} n+e_{i}} s_{n} \quad(n=0,1, \cdots),
$$

where $b_{i}, c_{i}, d_{i}$ and $e_{i}$ are all real with $\prod_{i=1}^{m} d_{i} \neq 0$ and $\prod_{i=1}^{m}\left(d_{i} n+e_{i}\right)$ $\neq 0$. Then

$$
\mu_{n} \rightarrow \prod_{i=1}^{m}\left(b_{i} / d_{i}\right) \sigma\left(A_{\lambda}\right)\left(\text { or }\left|A_{\lambda}\right|\right) \text { whenever } s_{n} \rightarrow \sigma\left(A_{\lambda}\right)\left(\text { or }\left|A_{\lambda}\right|\right) \text {. }
$$

Proof. That $s_{n} \rightarrow \sigma\left(A_{\lambda}\right) \quad\left(\right.$ or $\left.\left|A_{\lambda}\right|\right)$ implies $s_{n} /(n+k) \rightarrow 0 \quad\left(A_{\lambda}\right)$ (or $\left|A_{\lambda}\right|$ ), where $k$ is real, is known ([2] or [8]). It follows that

$$
\frac{b n+c}{d n+e} s_{n}=\frac{b}{d} s_{n}+\frac{c d-b e}{d^{2} n+d e} s_{n} \rightarrow \frac{b}{d} \sigma\left(A_{\lambda}\right)\left(\text { or }\left|A_{\lambda}\right|\right)
$$

for all real $b, c, d$ and $e$ with $d \neq 0$. The conclusion then follows immediately by repeatedly using the above result.

Define

$$
\nu_{n}=\frac{\Gamma(\alpha n+\beta+\lambda) \Gamma(n+1)}{\Gamma(\alpha n+\beta) \Gamma(n+\lambda+1)} s_{n} \quad \text { for } n=0,1, \cdots .
$$

We then have the following two lemmas: 
LEMMA 3. If $s_{n} \rightarrow \sigma(B, \alpha, \beta)$ and condition (C) holds, then for all $\lambda>-1, \nu_{n} \rightarrow \alpha^{\lambda} \sigma\left(A_{\lambda}\right)$.

LeMma 4. If $s_{n} \rightarrow \sigma|B, \alpha, \beta|$ and condition (C) holds, then for all $\lambda>-1, \nu_{n} \rightarrow \alpha^{\lambda} \sigma\left|A_{\lambda}\right|$.

Proof of Lemma 3. Let

$$
J(t)=\int_{0}^{\infty} e^{-u / t} u^{\lambda} S_{\alpha, \beta}(u) d u, \quad \text { where } 0<t<\infty .
$$

It follows by hypothesis that

$$
J(t)=\alpha \Gamma(\lambda+1) t^{\lambda+1}\left(\frac{t}{1+t}\right)^{\beta-1}\left(\frac{1+y}{1+t}\right)^{\lambda+1} \sigma_{\lambda}^{*}(y),
$$

where $t$ and $y$ are related by $(t /(1+t))^{\alpha}=y /(1+y)$, and

$$
\sigma_{\lambda}^{*}(y)=(1+y)^{-\lambda-1} \sum_{n=N}^{\infty} E_{n}^{\lambda} \nu_{n}\left(\frac{y}{1+y}\right)^{n} .
$$

Since $S_{\alpha, \beta}(u) \rightarrow \sigma$ as $u \rightarrow \infty$, it follows from Lemma 1 in [4] that

$$
J(t) / \Gamma(\lambda+1) t^{\lambda+1} \rightarrow \sigma \quad \text { as } t \rightarrow \infty .
$$

Furthermore $(1+y) /(1+t) \rightarrow 1 / \alpha$ as $y \rightarrow \infty$, and $t \rightarrow \infty$ if and only if $y \rightarrow \infty$. Hence

$$
\sigma_{\lambda}^{*}(y) \rightarrow \alpha^{\lambda} \sigma \quad \text { as } y \rightarrow \infty .
$$

This completes the proof.

Proof of Lemma 4. Let $J(t)$ and $\sigma_{\lambda}^{*}(y)$ be defined as in the proof of the above lemma. We see that

$$
\sigma_{\lambda}^{*}(y)=(1 / \alpha \Gamma(\lambda+1)) A_{1}(t) A_{2}(t) A_{3}(t),
$$

where

$$
A_{1}(t)=J(t) / t^{\lambda+1}, \quad A_{2}(t)=((1+t) / t)^{\beta-1}
$$

and

$$
A_{3}(t)=\left[\frac{1-(t /(1+t))^{\alpha}}{1-t /(1+t)}\right]^{\lambda+1}
$$

For $h>0$, it is easy to show that $A_{2}(t)$ and $A_{3}(t)$ are of bounded variation with respect to $t$ in $[h, \infty)$, whereas that $A_{1}(t)$ is of bounded variation in $[h, \infty)$ follows by hypothesis and Lemma 1. Thus $\sigma_{\lambda}^{*}(y)$ is of bounded variation with respect to $y$ in $[g, \infty)$, where $g$, greater 
than zero, is dependent on $h$. Thus this lemma follows from the definition and the previous lemma.

4. Theorems. All the following theorems are under the condition (C).

TheOREM 1. If $s_{n} \rightarrow \sigma(B, \alpha, \beta)$, then $s_{n} \rightarrow \sigma\left(A_{\lambda}\right)$ for all $\lambda>-1$.

Proof. Since $s_{n} \rightarrow \sigma\left(A_{\lambda}\right)$ implies $s_{n} \rightarrow \sigma\left(A_{\mu}\right)$ for all $\lambda>\mu>-1$ [2], we may assume that $\lambda$ is an integer, and so by Lemma 3,

$$
\nu_{n}=\frac{(\alpha n+\beta+\lambda-1)(\alpha n+\beta+\lambda-2) \cdots(\alpha n+\beta+1)}{(n+\lambda)(n+\lambda-1) \cdots(n+1)} s_{n} \rightarrow \alpha^{\lambda} \sigma\left(A_{\lambda}\right) .
$$

Therefore by Lemma $2, s_{n} \rightarrow \sigma\left(A_{\lambda}\right)$ for all $\lambda>-1$.

Theorem 2. If $s_{n} \rightarrow \sigma(B, \alpha, \beta)$, then $s_{n} \rightarrow \sigma\left[A_{\lambda}\right]_{p}$ for all $p \geqq 1, \lambda>-1$.

Proof. We need that [6], if $\lambda>-1$ and $p \geqq 1$, then $s_{n} \rightarrow \sigma\left[A_{\lambda}\right]_{p}$ if and only if $s_{n} \rightarrow \sigma\left(A_{\lambda}\right)$ and

$$
\int_{0}^{x}\left|y \frac{d}{d y} \sigma_{\lambda}(y)\right|^{p} d y=o(x), \quad \text { as } x \rightarrow \infty .
$$

Since

$$
y \frac{d}{d y} \sigma_{\lambda}(y)=(\lambda+1)\left[\sigma_{\lambda+1}(y)-\sigma_{\lambda}(y)\right]=o(y)
$$

as $y \rightarrow \infty$, by Theorem 1 . It follows immediately that

$$
\int_{0}^{x}\left|y \frac{d}{d y} \sigma_{\lambda}(y)\right|^{p} d y=o(x),
$$

as $x \rightarrow \infty$. This completes the proof of this theorem.

Since $s_{n} \rightarrow \sigma[B, \alpha, \beta]_{q}$ implies $s_{n} \rightarrow \sigma(B, \alpha, \beta)$ for all $q \geqq 1[3]$, the following is a corollary of the previous theorem.

TheOREM 3. If $s_{n} \rightarrow \sigma[B, \alpha, \beta]_{q}$, then $s_{n} \rightarrow \sigma\left[A_{\lambda}\right]_{p}$ for all $p \geqq 1$, $\lambda>-1, q \geqq 1$.

Finally, we give the corresponding result for absolute summability.

TheOREM 4. If $s_{n} \rightarrow \sigma|B, \alpha, \beta|$, then $s_{n} \rightarrow \sigma\left|A_{\lambda}\right|$ for all $\lambda>-1$.

Proof. Since $s_{n} \rightarrow \sigma\left|A_{\lambda}\right|$ implies $s_{n} \rightarrow \sigma\left|A_{\lambda+\delta}\right|$ for all $\delta>0$ [7], we may assume that $\lambda$ is an integer, and then the rest of the proof is similar to that of Theorem 1, except that Lemma 3 is replaced by Lemma 4. 
5. Remark. This section is presented for the sake of completeness. Let

$$
\begin{aligned}
A_{\alpha, \beta}(x) & =\int_{0}^{x} e^{-t} \sum_{n=N}^{\infty} \frac{a_{n} t^{\alpha n+\beta-1}}{\Gamma(\alpha n+\beta)} d t, \\
U_{\lambda}(y) & =\lambda \int_{0}^{y}(1+t)^{-\lambda-1} \sum_{n=0}^{\infty} E_{n}^{\lambda} a_{n}\left(\frac{t}{1+t}\right)^{n} d t .
\end{aligned}
$$

Both series are assumed to be convergent for all $t \geqq 0$. We note that the " $B$ " methods and the " $A$ " methods are obtained by formally replacing $S_{\alpha, \beta}(x)$ by $A_{\alpha, \beta}(x), \sigma_{\lambda}(y)$ by $U_{\lambda}(y)$ and $\sigma$ by $\sigma-s_{N-1}$; respectively.

The following results are known ([3] and [8] respectively).

5.1. $s_{n} \rightarrow \sigma\left(B^{\prime}, \alpha, \beta\right)$ (or $\left[B^{\prime}, \alpha, \beta\right]_{q}$, or $\left.\left|B^{\prime}, \alpha, \beta\right|\right)$ if, and only if, $s_{n} \rightarrow \sigma(B, \alpha, \beta+1)$ (or $[B, \alpha, \beta+1]_{q}$, or $\left.|B, \alpha, \beta+1|\right)$.

5.2. $s_{n} \rightarrow \sigma\left(A_{\lambda}\right)$ (or $\left[A_{\lambda}\right]_{p}$, or $\left.\left|A_{\lambda}\right|\right)$ if, and only if, $s_{n} \rightarrow \sigma\left(A_{\lambda-1}\right)$ (or $\left[A_{\lambda-1}\right]_{p}$, or $\left|A_{\lambda-1}\right|$ ).

With these, it is easy to show that in Theorems $1-4$, either $B$ or $A$, or both, may be replaced by $B^{\prime}$ or $A^{\prime}$, or both, respectively.

\section{REFERENCES}

1. D. Borwein, On methods of summability based on integral functions. II, Proc. Cambridge Philos. Soc. 56 (1960), 125-131. MR 22 \#6957.

2. - On a scale of Abel-type summability methods, Proc. Cambridge Philos. Soc. 53 (1957), 318-322. MR 19, 134.

3. D. Borwein and B. L. R. Shawyer, On Borel-type methods. I, II, Tôhoku Math. J. (2) 18 (1966), 283-298; ibid. (2) 19 (1967), 232-237; correction, 244. MR 35 \#3312; MR 35 \#7031; MR 36 \#6826.

4. G. Doetsch, Über den Zusammenhang zwischen Abelscher und Borelscher Summabilität, Math. Ann. 104 (1931), 403-414.

5. R. Jajte, On the compositions of integral means with Borel methods of summability Ann. Polon. Math. 14 (1963/64), 101-116. MR 28 \#2383.

6. B. P. Mishra, Strong summability of infinite series on a scale of Abel type summability methods, Proc. Cambridge Philos. Soc. 63 (1967), 119-127. MR 34 \#3156.

7. - Absolute summability of infinite series on a scale of Abel type summability methods, Proc. Cambridge Philos. Soc. 64 (1968), 377-387. MR 36 \#5564.

8. S. J. H. Rizvi, Abel-type summability, Ph.D. Thesis, Univ. of Western Ontario, 1969.

9. B. L. R. Shawyer, On the relation between the Abel and Borel-type methods of summability, Proc. Amer. Math. Soc. 22(1969), 15-19. MR 39 \#4551.

University of Western Ontario, London, Ontario, Canada 